\title{
Application of Data Visualization in Marine Data Platform
}

\author{
Xianjiu Guo ${ }^{1,}$, Yinghui Jiang ${ }^{1, b}$ \\ ${ }^{1}$ Dalian Ocean University, Dalian, China; \\ a879753691@qq.com, b1070713734@qq.com
}

This paper is supported by National Marine Public Welfare Projects (No.201205023-4).

\begin{abstract}
Keywords: Marine data; data visualization; component technology; database design
\end{abstract}
\begin{abstract}
Data visualization in marine data query system has an important significance for the improvement of data query efficiency and data friendly. Aiming at the problem of heterogeneous data in marine database, visual information database was designed, to provide a unified data layer interface for service layer. In addition, component technology was used to realize data conversion, data query and data visualization, in order to solve the limitation of general ocean data management system and to improve the scalability and portability of the system.
\end{abstract}

\section{Introduction}

Recently, Chinese marine management department carried out basic investigation, including the "908 Digital Ocean”, also carried out many other routine survey such as ocean stations, buoy and seabed. Many scattered databases with special functions were established. However, a comprehensive platform which can integrate all kinds of marine data library and visual display has not been researched, also, management and application of database should be studied further.

Many foreign institutions has established many data platforms with perfect functions. Currently, the USA National Oceanographic Data Center and Britain Oceanographic Data Center (BODC) has been able to achieve better management of marine data, and can realize search and query of marine data in a number of areas. Asia Pacific Data Center (APDRC) provided fast and convenient visualization for marine data with file format by using the grid-based LAS and DCHART server. Stanford University proposed Ozone integrated model to extend database ODMG. However, above system can only to carry out data visualization for data files with specific format, and visual display of database format is relatively weak. Also, this kind system is only designed for a specific data source, and is not suitable for all data source format. In addition, the system itself has complex structure and poor scalability.

This paper designed data visualization information database and visual components of marine data, to achieve unified management and reusability of the system, and map component was used to implement interactive query to realize visual display of marine data.

\section{System Structure}

Architecture. Marine data visualization system consists of data layer, business logic layer and application layer.

The data layer is the data source of marine elements of visualization, which is responsible for storage, read and write of ocean based data. It mainly includes meta database, basic database and information database. Meta database in charge of respective storage of data and attribute information. Effective management and high availability are achieved through retrieval and query of meta-data. Moreover, basic database contains marine hydrology, meteorology, physical oceanography, marine chemistry, marine biology and ecology, marine sediment, suspended matter, marine geophysics and Ocean Topography Basis, and each kind of database contains different categories of data. The design of each database is different, the data categories are various, and the amount of data is large, for these features, relative information of visualization is stored in visual information database, in order to provide a uniform interface for data visualization display and to improve system reusability. 


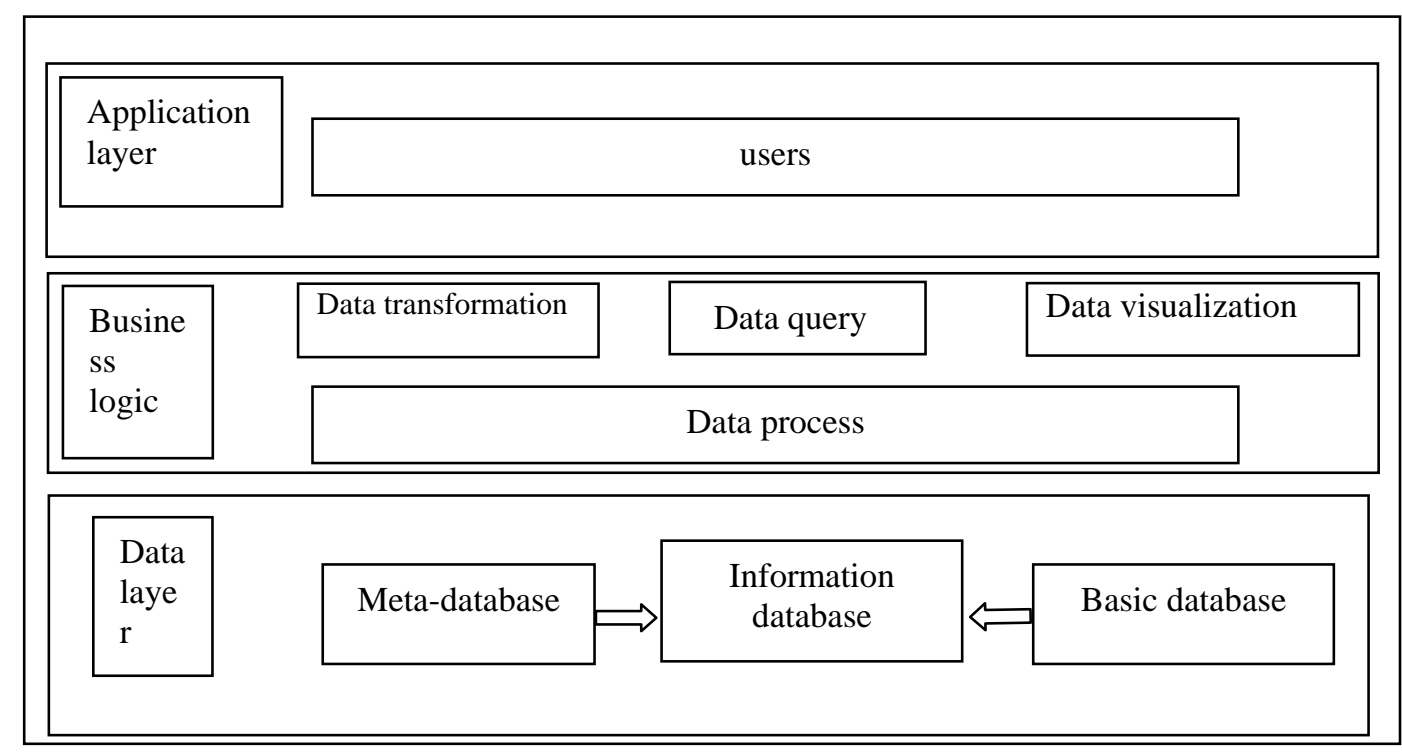

Fig.1 Architecture of Data Visualization

The business logic layer achieves all the business logic through the access to the data layer, including data processing module, data format conversion module, data query module and data visualization components.

The presentation layer display the results by using visual components according to the data obtained from logic layer in the way of curve graph.

Function Structure. Visualization system is composed by 3 function modules, and the concrete explanation is shown in figure 2.

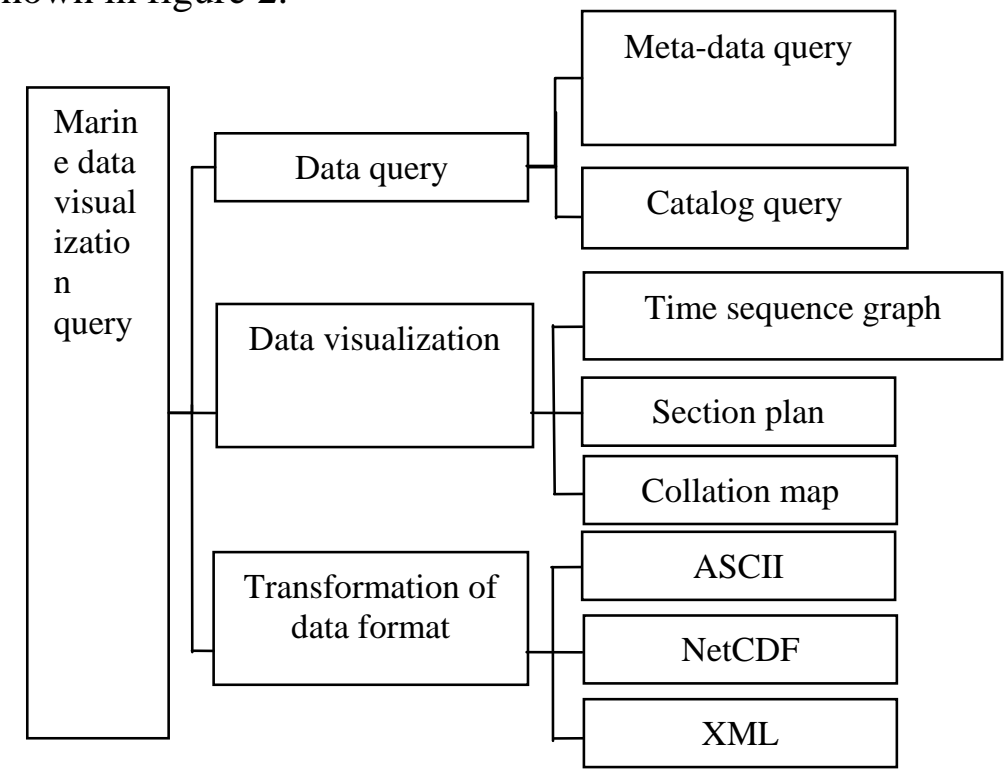

Fig.2 Diagram of System Functions

Visualization query and display system is divided into data format conversion, data query and data visualization.

(1) Data Format Conversion

The diversity of marine data should be displayed with different data form because of the varied storage format. Data conversion function converts database to the common storage format used for marine data, such as NetCDF, HDF, ASCII and commonly used XML standard format, in order to meet the diverse needs of users. 
(2) Data Query

Data query module includes meta-data based and directory based ways. Meta-data query refers to query meta-data meeting the conditions first, and the query data of database. The directory inquiry mode refers to classifying the data in database and to retrieve data according to discipline catalogue $^{[1-2]}$.

(3) Data Visualization

Data visualization refers to the users query data by map interactive mode, and display the data with curve view. Data visualization is a way of data query. Curve types include time sequence chart, section plan and collation map. Time curve displays the changes in the dimension of time of selected elements. Section plan is a data curves of a fixed point in time depth datum. Collation map denotes the comparison of the same element in multiple sites.

Data visualization is used to map information data to target view, and the mapping relation includes property definition and regulation, such as logic relation of information data, definition data value and knowledge layer mapping. Data expression of visual data is described as follows ${ }^{[3-4]}$ :

Data set $N=\left\{n_{i} \mid 1 \leq i \leq m\right\}$, $m$ is denoted as the number of mapping data

View set $V=\left\{v_{j} \mid 1 \leq j \leq m\right\}$, $m$ is the number of displayed views.

Set of data group $D G=\left\{d g_{k} \mid 1 \leq k \leq t\right\}$, data group is the set of data items of a view, and $t$ is the number of data group.

Data group $d g_{k}=\left\{d_{k h} \mid 1 \leq h \leq \varepsilon\right\}, \varepsilon$ is the number of data items of current data group.

\section{Design of Information Database}

Basic database of marine data includes marine hydrological and marine meteorological database. However, there are lots of differences among these data in terms of properties and structures. If we carry out visualization towards data of each class, large energy and time will be spent, as well as much unnecessary duplication of work will be produced. Moreover, and the system developed on this basis cannot be used in other databases due to poor expansibility and portability. Therefore, the design of database must consider universal and characteristics of marine data, to realize the unified access to the data source ${ }^{[5-6]}$.

Through the comparison and analysis of the data in various databases, we found that although data structure of each database is different, each database contains spatio-temporal information, attribute information and the characteristic elements required by visualization. Such information will be individually extracted and rebuild a new database, that is, visual information database, to record data categories, visual elements, set time range, latitude and longitude range, source of data and curve type information, through which to retrieve the data of basic database.

\section{Conclusion}

This paper designed visual information database, shielded the underlying heterogeneous database and standardized the data of data layer. In addition, business logic layer can access the data of each database only through an interface, which laid the foundation for the unified data processing.

\section{References}

[1] Serra-Sogas N,O Hara P D,Canessa R,et al. Visualization of spatial patterns and temporal trends for aerial surveillance of illegal oil discharges in western Canadian marine waters[J]. Marine Pollution Bulletin, 2011,56(5):825-833 
[2] Kulawiak M,Prospathopoulos A,Perivoliotis L,et al. Interactive visualization of marine pollution monitoring and forecasting data via a Web-basedGIS[J]. Computers \&amp; Geosciences, 2010,36(8):1069-1080

[3] Hader D P. Novel Method to determine vertical distributions of phytoplankton in marine water columns[J]. Environmental and Experimental Botany,2010,35(4):547-555.

[4] Pech-Pacheco J L,Alvarez-Borrego J. Optical-digital system applied to the identification of five phytoplankton species[J]. Marine Biology, 2011(132):357-365.

[5] Tang X, Stewart W K, Huang H, et al. Automatic plankton image recognition[J]. Artificial Intelligence Review, 2011,12:177-199.

[6] Buf H, Bayer M M. Automatic Diatom Identification[J]. Singapore;World Scientific, 2012. 